\title{
Linearization Modeling for Non-smooth Dynamical Systems with Approximated Scalar Sign Function
}

\author{
Jian Zhang, Yongpeng Zhang, Member, IEEE, Warsame Ali, Leang-san Shieh, Senior Member, IEEE
}

\begin{abstract}
Traditionally, one typical way to deal with the sign function constrained non-smooth system is to divide the state space into two or more subspaces based on the direction of sign function. The resulting piecewise model is with complicated structure, leading to the difficulty in controller design as well as stability analysis. In this paper, an innovative concept of approximated scalar sign function is introduced. Through the proposed methodology, the non-smooth dynamical system model can be transformed into a universal and smooth model. Thus, the following optimal linearization can be applied to obtain the local linear model at any operating point. Finally, two illustrative examples, representing hysteresis and friction, respectively, are given to demonstrate the effectiveness of the proposed method.
\end{abstract}

\section{INTRODUCTION}

A particular non-smooth dynamical model is with sign function constrained nonlinearity, which is widely existing in models of hysteresis, friction, backlash, etc. Traditionally, one typical way to deal with such a nonsmooth nonlinear system is to divide the state space into two or more subspaces based on the direction of sign function, and then make the controller design for each subspace [1] [2]. This approach has several disadvantages: (i) The piecewise functions are with complicated structure, leading to the difficulty in controller design as well as stability analysis; (ii) Since there exist several subspaces, the local controllers need to be triggered at the boundary crossing. This requires a high sampling rate, leading to a heavy or even impractical computational burden in real-time; (iii) If the non-smooth nonlinear system is crossing coupled, of high order, with state constraint, etc, the problem solving with the traditional piecewise approach will be very challenging.

In this paper, an innovative idea is introduced to utilize an approximated scalar sign function to transform the nonsmooth nonlinear model into a universal and smooth model. This method stems from previous research works on the matrix sign function and the matrix sector function [3] [4]. The proposed scalar sign function is the counterpart of the

Manuscript received March 2, 2011. This work was supported by National Science Foundation under Grant 0942807 and 0817462.

J. Zhang and W. Ali are with the Electrical \& Computer Engineering Department, Prairie View A\&M University, Prairie View, TX 77446 USA (e-mail: jzhang1@pvamu.edu,whali@pvamu.edu).

Y. Zhang is with the Engineering Technology Department, Prairie View A\&M University, Prairie View, TX 77446 USA (phone: 936-261-9869; fax: 936-261-9867; e-mail: ypzhang@pvamu.edu).

L. Shieh is with the Electrical \& Computer Engineering Department, University of Houston, Houston, TX 77064 USA (e-mail: 1shieh@uh.edu). matrix sign function. In the development of sign function, a continued fraction expansion form was established. It was shown that a certain order of truncation of the expansion can effectively approximate the sign function. Through the proposed approximated scalar sign function, the non-smooth dynamical model can be transformed into a smooth function. Then the following optimal linearization can be conveniently applied.

The rest of this paper is organized as follows. Section II describes the development of approximated scalar sign function. Section III introduces the optimal linearization method for the resulting smooth system model. In Section IV, two illustrative examples, representing coulomb friction and Bouc-Wen hysteresis, respectively, are given to demonstrate the effectiveness of the proposed modeling method. Section $\mathrm{V}$ concludes this paper.

\section{APPROXIMATED SCALAR SIGN FUNCTION}

The scalar sign function is defined in [3] as

$$
\operatorname{sign}(z)= \begin{cases}1 & \text { if } \operatorname{Re}(z)>0 \\ -1 & \text { if } \operatorname{Re}(z)<0\end{cases}
$$

where $z \in C^{+} \cup C^{-}, C^{+}$and $C^{-}$denotes the open right-half complex plane and the open left-half complex plane, respectively. It is noted that $\operatorname{sign}(z)$ is undefined for $\operatorname{Re}(z)=0$.

An alternative form of the scalar sign function is presented in [5] as

$$
\operatorname{sign}(z)=z / \sqrt{z^{2}},
$$

where $z \in C^{+} \cup C^{-}$and

$$
\sqrt{z^{2}}= \begin{cases}z & \text { if } \operatorname{Re}(z)>0 \\ -z & \text { if } \operatorname{Re}(z)<0,\end{cases}
$$

which can be expressed by the continued fraction expansion form as

$$
\sqrt{z^{2}}=1+\frac{z^{2}-1}{2+\frac{z^{2}-1}{2+\cdots}} .
$$

The $j$-th truncation of ( $3 \mathrm{~b})$ can be expressed as

$$
\left(\sqrt{z^{2}}\right)_{j}=z \frac{(1+z)^{j}+(1-z)^{j}}{(1+z)^{j}-(1-z)^{j}} \text {, for } j=1,2, \ldots
$$


It can be shown that

$$
\sqrt{z^{2}}=\lim _{j \rightarrow \infty}\left(\sqrt{z^{2}}\right)_{j}
$$

where $j$ is known as the approximation order.

Substituting (3c) into (2) yields the approximated scalar sign function

$$
\operatorname{sign}_{j}(z)=\frac{(1+z)^{j}-(1-z)^{j}}{(1+z)^{j}+(1-z)^{j}} .
$$

And

$$
\operatorname{sign}(z)=\lim _{j \rightarrow \infty} \operatorname{sign}_{j}(z) \quad \text { for } z \in C^{+} \cup C^{-} .
$$

Additionally, $\lim _{j \rightarrow \infty} \operatorname{sign}(z)=0$ for $\operatorname{Re}(z)=0$. Therefore, the definition of scalar sign function (1) can be extended to the whole complex plane $z \in C$ as

$$
\operatorname{sign}(z)=\lim _{j \rightarrow \infty} \operatorname{sign}_{j}(z)= \begin{cases}1 & \text { if } \operatorname{Re}(z)>0 \\ 0 & \text { if } \operatorname{Re}(z)=0 \\ -1 & \text { if } \operatorname{Re}(z)<0 .\end{cases}
$$

Thus, the scalar sign function for a real number can be specified as

$$
\operatorname{sign}(\sigma)=\lim _{j \rightarrow \infty} \operatorname{sign}_{j}(\sigma)= \begin{cases}1 & \text { if } \sigma>0 \\ 0 & \text { if } \sigma=0 \\ -1 & \text { if } \sigma<0 .\end{cases}
$$

where $\sigma \in R$, and $j$-th order truncated approximation for (8) can be given as

$$
\operatorname{sign}_{j}(\sigma)=\frac{(1+\sigma)^{j}-(1-\sigma)^{j}}{(1+\sigma)^{j}+(1-\sigma)^{j}} .
$$

Differentiating (9) with respect to $\sigma$ yields

$$
\frac{d\left(\operatorname{sign}_{j}(\sigma)\right)}{d \sigma}=\frac{2 j\left[(1-\sigma)^{j-1}(1+\sigma)^{j}+(1+\sigma)^{j-1}(1-\sigma)^{j}\right]}{\left[(1+\sigma)^{j}+(1-\sigma)^{j}\right]^{2}} \text {. }
$$

Fig. 1(a) shows the value of $\operatorname{sign}_{j}(\sigma)$ defined in (9) for $\sigma \in[-0.5,0.5]$ with the step size of 0.01 and Fig. 1(b) is for $\sigma \in[-10,10]$ with the step size of 1 . The derivative value given by (10) for $\sigma \in[-0.5,0.5]$ is shown in Fig. 2 . It can be seen from Fig. 1 that the higher the approximation order $j$, the closer the approximated scalar sign function (9) to approach the original sign function (8). From Fig. 2, it is shown that the approximated scalar sign function (9) is differentiable everywhere with the largest derivative value at $\sigma=0$, which equals the approximation order $j$.

For a non-smooth dynamical system with sign function constraint, a universal and smooth model can be obtained by replacing (8) with the proposed approximated scalar sign function (9). Thus a universal linearization can be conducted, so that traditional piecewise models can be avoided. .

Remark1: Absolute value function can also be represented by the proposed approximated scalar sign function as $|x|=x \cdot \operatorname{sign}(x) \approx x \cdot \operatorname{sign}_{j}(x)$.

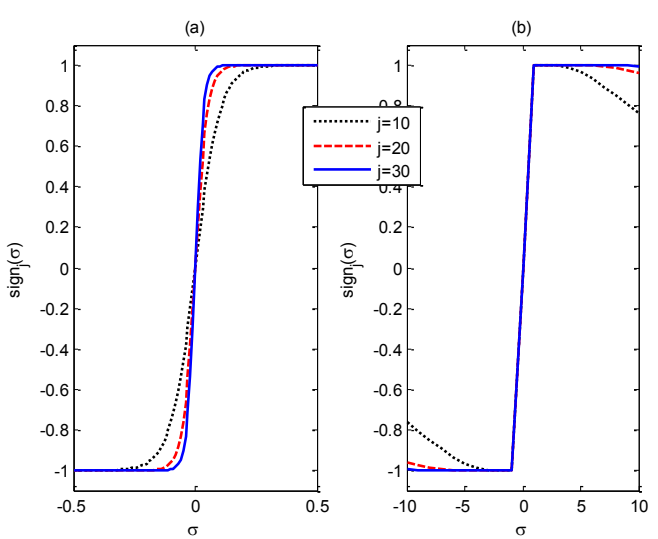

Fig. 1. Proposed approximated scalar sign function (9) with different approximation order.

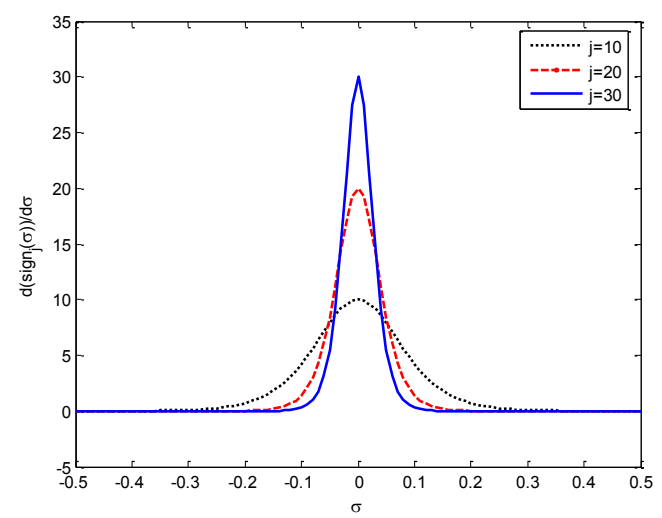

Fig. 2. Differentiation (10) of approximated scalar sign function with different approximation order.

\section{OPTIMAL LINEARIZATION}

With a smooth nonlinear model available, the next step is to conduct optimal linearization to obtain the local linear model at any operating point of interest. Consider a general class of smooth nonlinear system in the form

$$
\begin{aligned}
& x^{\prime}(t)=f(x(t))+G(x(t)) u(t) \\
& y(t)=C x(t)
\end{aligned}
$$

where $x(t) \in \mathfrak{R}^{n}$ is the state vector, $u(t) \in \mathfrak{R}^{m}$ is the input vector, $y(t) \in \mathfrak{R}^{p}$ is the output vector, $f(\cdot): \mathfrak{R}^{n} \rightarrow \mathfrak{R}^{n}$ with $f(0)=0$ and $G(\cdot): \mathfrak{R}^{m} \rightarrow \mathfrak{R}^{n}$ are both smooth nonlinear functions, $C \in \mathfrak{R}^{p \times n}$ is a constant matrix. Local linearization is to find a local linear model at an operating point $x_{k}(t)$, which is not the $k$-th component of $x(t)$, in the form

$$
x^{\prime}(t)=A_{k} x(t)+B_{k} u(t)
$$

where $A_{k}$ and $B_{k}$ are constant matrices of appropriate dimensions. If this linear model have the similar dynamics as the nonlinear system around the operating point of interest, then mature control theory and numerous design techniques for linear systems can be taken advantage to yield a functional controller for the nonlinear system. 
Based on Taylor expansion, Jacobian linearization [6] is a commonly used method to make local linearization. However, as commented in [7] and [8], truncated Taylor expansion often results in an affine rather than linear model in $x$ and $u$, even if the operating point is an equilibrium. The only exception is the trivial case that the operating point is just at the equilibrium of the origin. These comments can be confirmed with an autonomous nonlinear system

$$
x^{\prime}(t)=f(x(t))
$$

where $f(x(t))$ is a scalar nonlinear function. At an arbitrary operating point $x_{k}$, the resulting linear model of (13) from truncated Taylor expansion is

$$
x^{\prime}=f\left(x_{k}\right)+\left.\frac{d f}{d x}\right|_{x=x_{k}}\left(x-x_{k}\right)=\left.\frac{d f}{d x}\right|_{x=x_{k}} x+f\left(x_{k}\right)-\left.\frac{d f}{d x}\right|_{x=x_{k}} x_{k}
$$

which describes a tangent line $\mathrm{T}$ to the curve $f(x)$ through the operating point in Fig. 3. Since the line $\mathrm{T}$ is not crossing the origin, the intercept on $x^{\prime}$ will lead to an affine model. The desired linear model (12) for the system (13) is a straight line crossing both the origin and the operating point, shown as the line $\mathrm{L}$ in Fig. 3. The only exception for the tangent line $\mathrm{T}$ to go through the origin is the operating point $x_{k}=0$ with $f(0)=0$.

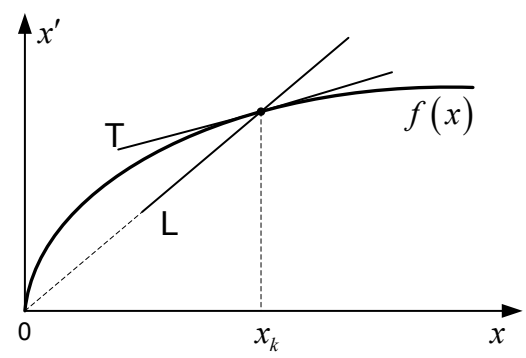

Fig. 3. Comparison of an affine model and a linear model.

To overcome the weakness of Jacobian linearization, Teixeira and Zak proposed an optimal linearization method in [7]. According to this method, an optimal linear model (12) can be obtained at any operating point, which has the exact dynamics of the nonlinear system (11a) at the operating point and minimum approximation error (in the least square sense) in the vicinity of the operating point. The reasoning process is briefed below.

In order to well approximate the dynamics of (11a) in the vicinity of the operating point $x_{k}, A_{k}$ and $B_{k}$ in (12) should satisfy that in a neighborhood of $x_{k}$,

$$
f(x)+G(x) u \approx A_{k} x+B_{k} u \text { for any } u
$$

and

$$
f\left(x_{k}\right)+G\left(x_{k}\right) u=A_{k} x_{k}+B_{k} u \text { for any } u .
$$

Since the control input $u$ can be arbitrary, it is necessary that

$$
G\left(x_{k}\right)=B_{k} \text {. }
$$

Then (15) becomes

$$
f(x) \approx A_{k} x
$$

and

$$
f\left(x_{k}\right)=A_{k} x_{k}
$$

namely,

$$
f_{i}(x) \approx a_{k}^{i} x
$$

and

$$
f_{i}\left(x_{k}\right)=a_{k}^{i} x_{k},
$$

where $f_{i}(\cdot): \mathfrak{R}^{n} \rightarrow \mathfrak{R}$ is the $i$-th component of $f, a_{k}^{i}$ is the $i$-th row of $A_{k}, i=1,2, \ldots, n$. Then applying truncated Taylor expansion about $x_{k}$ to $f_{i}(x)$ in (18a) produces

$$
f_{i}\left(x_{k}\right)+\nabla f_{i}\left(x_{k}\right)\left(x-x_{k}\right) \approx a_{k}^{i} x
$$

where $\nabla f_{i}\left(x_{k}\right): \mathfrak{R}^{n} \rightarrow \mathfrak{R}^{n}$ is the gradient vector of $f_{i}$ evaluated at $x_{k}$. Substituting (18b) into (19), it is obtained

$$
\nabla f_{i}\left(x_{k}\right)\left(x-x_{k}\right) \approx a_{k}^{i}\left(x-x_{k}\right) .
$$

Then the problem of (15) is reduced to find $a_{k}^{i}$ such that it is 'as close as possible' to $\nabla f_{i}\left(x_{k}\right)$ with $a_{k}^{i} x_{k}=f_{i}\left(x_{k}\right)$. For this purpose, a constrained minimization problem is formulated as

$$
\min E:=\frac{1}{2}\left\|\nabla f_{i}\left(x_{k}\right)-a_{k}^{i}\right\|_{2}^{2} \text { subject to } a_{k}^{i} x_{k}=f_{i}\left(x_{k}\right) .
$$

As a convex constrained optimization problem, (21) has the solution

$$
\begin{aligned}
& \nabla_{a_{k}^{i}} E+\lambda \nabla_{a_{k}^{i}}\left(a_{k}^{i} x_{k}-f_{i}\left(x_{k}\right)\right)=0 \\
& a_{k}^{i} x_{k}=f_{i}\left(x_{k}\right)
\end{aligned}
$$

where $\nabla_{a_{k}^{i}}$ indicates that the gradient is taken with respect to $a_{k}^{i}$ and $\lambda$ is the Lagrange multiplier. By taking differentiations, (22a) is solved as

$$
a_{k}^{i}-\nabla f_{i}\left(x_{k}\right)+\lambda x_{k}^{T}=0
$$

where $x_{k}^{T}$ is the transpose of $x_{k}$. When $x_{k} \neq 0$, rightmultiplying two sides of (23) by $x_{k}$ and using (22b) can solve $\lambda$ as

$$
\lambda=\frac{\nabla f_{i}\left(x_{k}\right) x_{k}-f_{i}\left(x_{k}\right)}{\left\|x_{k}\right\|_{2}^{2}}
$$

where $\left\|x_{k}\right\|_{2}$ is the Euclidean norm of $x_{k}$. Substituting (24) into (23) gives

$$
a_{k}^{i}=\nabla f_{i}\left(x_{k}\right)+\frac{f_{i}\left(x_{k}\right)-\nabla f_{i}\left(x_{k}\right) x_{k}}{\left\|x_{k}\right\|_{2}^{2}} x_{k}{ }^{T} .
$$

When $x_{k}=0, a_{k}^{i}$ can be solved directly from (23) as

$$
a_{k}^{i}=\nabla f_{i}\left(x_{k}\right) \text {. }
$$

Collecting (16) and (25) gives $A_{k}$ and $B_{k}$ in (12) as

$$
\begin{array}{ll}
A_{k}= \begin{cases}\nabla f\left(x_{k}\right)+\frac{f\left(x_{k}\right)-\nabla f\left(x_{k}\right) x_{k}}{\left\|x_{k}\right\|_{2}^{2}} x_{k}^{T} & \text { for } x_{k} \neq 0 \\
\nabla f(0) & \text { for } x_{k}=0\end{cases} \\
B_{k}=G\left(x_{k}\right),
\end{array}
$$


where $\nabla f\left(x_{k}\right)$ is the Jacobian matrix of $f(x)$ evaluated at the operating point $x_{k}$. It is noted that the case for $x_{k}=0$ in (26) agrees with the exception case mentioned before, that the operating point happens to be the equilibrium of the origin.

Remark2: When $f(x)$ in (11a) is a scalar nonlinear function, (26) is reduced to a scalar number as

$$
A_{k}= \begin{cases}f\left(x_{k}\right) / x_{k} & \text { for } x_{k} \neq 0 \\ f^{\prime}(0) & \text { for } x_{k}=0\end{cases}
$$

\section{ILLUSTRATIVE EXAMPLES}

\section{A. Coulomb Friction in DC Motor}

Widely existing in mechanical systems, friction can potentially cause steady-state error, limit cycle, or stick-slip phenomenon especially at a low speed in the conventional linear control of positioning systems [9]. This example focuses on a simple case of coulomb friction occurring in DC motor. Coulomb friction is usually ignored in the formulation of DC motor's state-space model and regarded as a disturbance in conventional control designs. It is a good example to illustrate the effectiveness of the proposed method and presents an innovative way to include the friction in system modeling.

Considering coulomb friction, DC motor system is given as

$$
\begin{aligned}
& V_{m}=R_{a} i+L_{a} i^{\prime}+K_{m} \omega \\
& K_{t} i=T_{L}+T_{f}+J \omega^{\prime}+B \omega
\end{aligned}
$$

where $i$ is the motor current, $\omega$ is the motor speed, $V_{m}$ is the motor voltage, $T_{L}$ and $T_{f}$ are the load torque and coulomb friction, respectively; other physical parameters are explained in Table I. The commonly used DC motor model in the state-space form is

$$
\left(\begin{array}{l}
i^{\prime} \\
\omega^{\prime}
\end{array}\right)=\left[\begin{array}{cc}
-R_{a} / L_{a} & -K_{m} / L_{a} \\
K_{t} / J & -B / J
\end{array}\right]\left(\begin{array}{l}
i \\
\omega
\end{array}\right)+\left(\begin{array}{c}
1 / L_{a} \\
0
\end{array}\right) V_{m}
$$

where the load torque $T_{L}$ and the coulomb friction $T_{f}$ in (28b)

\begin{tabular}{|c|c|c|c|}
\hline Symbol & Description & Value & Unit \\
\hline$R_{a}$ & Motor armature resistance & 0.764 & $\Omega$ \\
\hline$L_{a}$ & Motor inductance & 0.0026 & $\mathrm{H}$ \\
\hline$K_{t}$ & Motor torque constant & 0.0805 & $\mathrm{~N} \cdot \mathrm{m}$ \\
\hline$K_{m}$ & $\begin{array}{l}\text { Motor back-electromotive force } \\
\text { constant }\end{array}$ & 0.0805 & $\mathrm{~V} /(\mathrm{rad} / \mathrm{s})$ \\
\hline$B$ & Viscous damping constant & 0 & $\mathrm{~N} \cdot \mathrm{m} \cdot \mathrm{s}$ \\
\hline$J$ & Equivalent moment of inertia & 0.0022 & $\mathrm{~kg} \cdot \mathrm{m}^{2}$ \\
\hline$\mu$ & Coulomb friction constant & 0.033 & $\mathrm{~N} \cdot \mathrm{m}$ \\
\hline
\end{tabular}
are ignored.

TABLE I

DC MOTOR PARAMETER

Not like the load torque $T_{L}$ which is an external variable, the coulomb friction $T_{f}$ is an intrinsic element which can be identified through characterization experiment. Depending on the direction of rotation, the coulomb friction $T_{f}$ can be modeled as

$$
T_{f}=\mu \cdot \operatorname{sign}(\omega)
$$

where $\mu$ is the coulomb friction constant given in Table I and $\operatorname{sign}(\omega)$ is the sign of speed, denoting the rotation direction.

Obviously, substituting (30) into (28) will give a non-smooth DC motor model.

By replacing $\operatorname{sign}(\omega)$ with the proposed approximated scalar sign function (9), the non-smooth coulomb friction (30) becomes a smooth rational function

$$
T_{f} \approx \mu \cdot \operatorname{sign}_{j}(\omega)
$$

where $\operatorname{sign}_{j}(\omega)$ is given by (9). This smooth function is a scalar nonlinear function of $\omega$, and then optimal linearization can be given by Remark2 as

$$
T_{f} \approx K_{f} \omega
$$

where

$$
K_{f}= \begin{cases}\mu \frac{\operatorname{sign}_{j}\left(\omega_{k}\right)}{\omega_{k}} & \text { for } \omega_{k} \neq 0 \\ \left.\mu \frac{d\left(\operatorname{sign}_{j}(\omega)\right)}{d \omega}\right|_{\omega_{k}=0}=\mu j & \text { for } \omega_{k}=0\end{cases}
$$

in which $\omega_{k}$ is the motor speed at the operating point and $\left.\frac{d\left(\operatorname{sign}_{j}(\omega)\right)}{d \omega}\right|_{\omega_{k}=0}$ is the differentiation (10) evaluated at $\omega=0$, which equals the approximation order $j$.

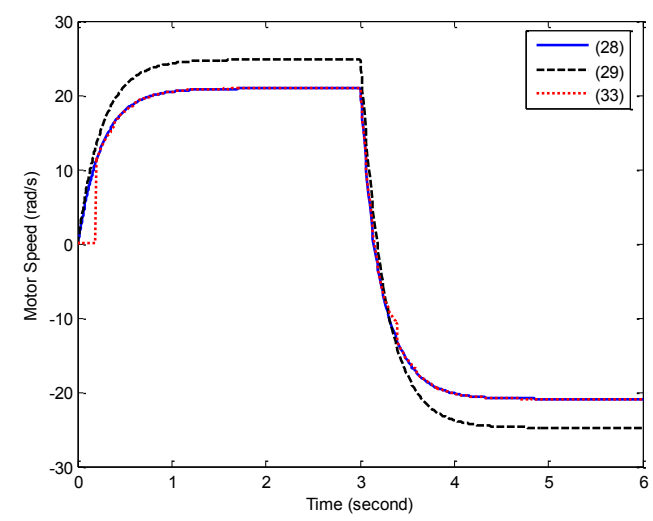

Fig. 4. Comparison of open loop controls of (28) and (33).

Substituting (32) into (28) and ignoring external load torque $T_{L}$ yields the linear DC motor model in the state-space form as

$$
\left(\begin{array}{l}
i^{\prime} \\
\omega^{\prime}
\end{array}\right)=\left[\begin{array}{cc}
-R_{a} / L_{a} & -K_{m} / L_{a} \\
K_{t} / J & -\left(B+K_{f}\right) / J
\end{array}\right]\left(\begin{array}{l}
i \\
\omega
\end{array}\right)+\left(\begin{array}{c}
1 / L_{a} \\
0
\end{array}\right) V_{m}
$$

where $K_{f}$ accounts for the coulomb friction $T_{f}$. In order to reflect the dynamic properties at different operating point, this model needs to be updated periodically. Fig. 4 shows the simulation results of open loop control of three models: the non-smooth DC motor model (28) with coulomb friction (30), the conventional linear DC motor model (29) which 
does not include coulomb friction, and the proposed linear DC motor model (33) which is updated periodically to account for the coulomb friction. Setting the motor voltage $V_{m}$ as $2 \mathrm{~V}$ for the first $3 \mathrm{~s}$, and then changed to $-2 \mathrm{~V}$, the load torque $T_{L}=0$, and the proposed model (33) with the approximation order $j=50$ is updated at the sampling period of $0.2 \mathrm{~s}$. It can be seen that except for the first beginning, the speed curve of proposed model (33) quickly converge to the original non-smooth model (28), while the curve made of conventional model (29) which does not include friction departs from the actual system performance.

\section{B. Bouc-Wen Hysteresis}

Hysteresis refers to the input-output dynamic relations having memory effects, which is encountered in many physical systems, like electronics, magnetism, mechanics, and structures, etc. Introduced by Bouc in [10] and extended by Wen in [11], Bouc-Wen model was originally used to describe the hysteretic relation between the restoring force and the displacement. Over the past decades, this analytic model has been extensively used in modeling a variety of hysteretic patterns. A comprehensive survey on Bouc-Wen model and its applications can be found in [12] [13].

This example considers a single-degree-of-freedom system

$$
m s^{\prime \prime}+c s^{\prime}+R(t)=e(t)
$$

where $s$ is the displacement, $R(t)$ is the restoring force, $e(t)$ is an excitation force, $m$ is the mass and $c$ is the viscous damping constant. According to the Bouc-Wen model in [11], the restoring force $R(t)$ is described by

$$
R(t)=\alpha k s(t)+(1-\alpha) D k h(t)
$$

where $\alpha, k, D$ are physical parameters related to stiffness, elasticity etc; $h$ is a hysteretic variable, usually called the hysteretic displacement, that follows the following nonsmooth differential equation

$$
h^{\prime}=D^{-1}\left(\lambda s^{\prime}-\beta\left|s^{\prime}\right||h|^{n-1} h-\gamma s^{\prime}|h|^{n}\right)
$$

where $\lambda, \beta, \gamma$ and $n$ are dimensionless quantities shaping the hysteretic behavior. Equation (36) can be also expressed as

$$
h^{\prime}= \begin{cases}D^{-1}\left(\lambda s^{\prime}-\beta\left|s^{\prime}\right| h^{n}-\gamma s^{\prime}|h|^{n}\right) & \text { for } n \text { is odd } \\ D^{-1}\left(\lambda s^{\prime}-\beta\left|s^{\prime}\right| h^{n-1}|h|-\gamma s^{\prime} h^{n}\right) & \text { for } n \text { is even. }\end{cases}
$$

Taking $\left(s, s^{\prime}, h\right)^{T}$ as the state $x$, the hysteretic singledegree-of-freedom system (34)-(36) can be reformulated as

$$
x^{\prime}=f(x)+B e(t)
$$

where

$$
f(x)=\left\{\begin{array}{l}
s^{\prime} \\
-\frac{\alpha k}{m} s-\frac{c}{m} s^{\prime}-\frac{(1-\alpha) D k}{m} h \\
D^{-1}\left(\lambda s^{\prime}-\beta\left|s^{\prime}\right||h|^{n-1} h-\gamma s^{\prime}|h|^{n}\right),
\end{array} \quad B=\left(\begin{array}{c}
0 \\
\frac{1}{m} \\
0
\end{array}\right) .\right.
$$

For a local linear model, since $B$ is constant, only state matrix $A_{k}$ of (38) is needed at any operating point. However, this hysteretic system (38) is a non-smooth system due to the absolute value functions in $f(x)$, so the optimal linearization method cannot be applied directly.

By Remark1, the absolute function in $f(x)$ can be transformed into the form of sign function. Then following the proposed method, the sign functions are replaced with the approximated scalar sign function (9), thus resulting in an approximate smooth function $f_{j}(x)$. For space saving purpose, only the case that $n$ is even in (37) is discussed in this paper. The case that $n$ is odd can be treated in the same way. When $n$ is even, the approximate smooth function $f_{j}(x)$ is obtained as

$$
f_{j}(x)=\left\{\begin{array}{l}
s^{\prime} \\
-\frac{\alpha k}{m} s-\frac{c}{m} s^{\prime}-\frac{(1-\alpha) D k}{m} h \\
D^{-1}\left\{\lambda s^{\prime}-\beta s^{\prime}\left[\operatorname{sign}_{j}\left(s^{\prime}\right)\right] h^{n}\left[\operatorname{sign}_{j}(h)\right]-\gamma s^{\prime} h^{n}\right\}
\end{array}\right.
$$

where $\operatorname{sign}_{j}(\cdot)$ is given by (9). The Jacobian matrix of $f_{j}(x)$ is

$$
\nabla f_{j}(x)=\left[\begin{array}{ccc}
0 & 1 & 0 \\
-\frac{\alpha k}{m} & -\frac{c}{m} & -\frac{(1-\alpha) D k}{m} \\
0 & D_{32} & D_{33}
\end{array}\right]
$$

where

$$
\begin{gathered}
D_{32}=D^{-1}\left\{\lambda-\beta\left[\operatorname{sign}_{j}\left(s^{\prime}\right)\right] h^{n}\left[\operatorname{sign}_{j}(h)\right]\right. \\
\left.-\beta s^{\prime} \frac{d\left(\operatorname{sign}_{j}\left(s^{\prime}\right)\right)}{d\left(s^{\prime}\right)} h^{n}\left[\operatorname{sign}_{j}(h)\right]-\gamma h^{n}\right\} \\
D_{33}=D^{-1}\left\{-\beta s^{\prime}\left[\operatorname{sign}_{j}\left(s^{\prime}\right)\right] n h^{n-1}\left[\operatorname{sign}_{j}(h)\right]\right. \\
\left.-\beta s^{\prime}\left[\operatorname{sigh}_{j}\left(s^{\prime}\right)\right] h^{n} \frac{d\left(\operatorname{sign}_{j}(h)\right)}{d h}-\gamma s^{\prime} n h^{n-1}\right\}
\end{gathered}
$$

in which $\frac{d\left(\operatorname{sign}_{j}(\cdot)\right)}{d(\cdot)}$ is shown in (10). At the equilibrium point $x=0,(40)$ is reduced to

$$
\nabla f_{j}(0)=\left[\begin{array}{ccc}
0 & 1 & 0 \\
-\frac{\alpha k}{m} & -\frac{c}{m} & -\frac{(1-\alpha) D k}{m} \\
0 & \frac{\lambda}{D} & 0
\end{array}\right] .
$$

Evaluating (39) and (40) at the operating point $x_{k}=\left(s_{k}, s_{k}^{\prime}, h_{k}\right)^{T} \neq 0$ and substituting the results into (26) yields the state matrix of optimal linear model as

$$
A_{k}=\nabla f_{j}\left(x_{k}\right)+\frac{f_{j}\left(x_{k}\right)-\nabla f_{j}\left(x_{k}\right) x_{k}}{\left\|x_{k}\right\|_{2}^{2}} x_{k}^{T}
$$




$$
=\left[\begin{array}{ccc}
0 & 1 & 0 \\
-\frac{\alpha k}{m} & -\frac{c}{m} & -\frac{(1-\alpha) D k}{m} \\
\Phi_{k} s_{k} & \left.D_{32}\right|_{x=x_{k}}+\Phi_{k} s_{k}^{\prime} & \left.D_{33}\right|_{x=x_{k}}+\Phi_{k} h_{k}
\end{array}\right]
$$

where $\left.D_{32}\right|_{x=x_{k}}$ and $\left.D_{33}\right|_{x=x_{k}}$ are $D_{32}$ and $D_{33}$ evaluated at the operating point $x_{k}$, respectively;

$$
\begin{aligned}
\Phi_{k}=\left\{D^{-1}\left[\lambda s_{k}^{\prime}-\beta s_{k}^{\prime} \cdot \operatorname{sign}_{j}\left(s_{k}^{\prime}\right) \cdot h_{k}{ }^{n} \cdot \operatorname{sign}_{j}\left(h_{k}\right)-\gamma s_{k}^{\prime} h_{k}{ }^{n}\right]\right. \\
\left.-\left.D_{32}\right|_{x=x_{k}} s_{k}^{\prime}-\left.D_{33}\right|_{x=x_{k}} h_{k}\right\} /\left(s_{k}{ }^{2}+s_{k}^{\prime 2}+h_{k}{ }^{2}\right) .
\end{aligned}
$$

By utilizing the approximated scalar sign function (9) and optimal linearization (26), the optimal linear model of hysteretic single-degree-of-freedom system (38) can be found in the state-space form as

$$
x^{\prime}(t)=A_{k} x(t)+B e(t)
$$

where $A_{k}$ is given by (41) at the equilibrium point or by (42) at off-equilibrium points, and $B=(0,1 / \mathrm{m}, 0)^{T}$.

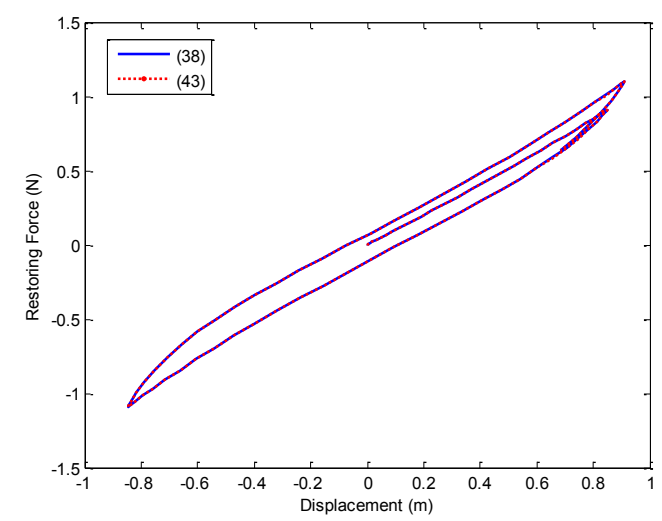

Fig. 5. Hysteresis loops of (38) and (43).

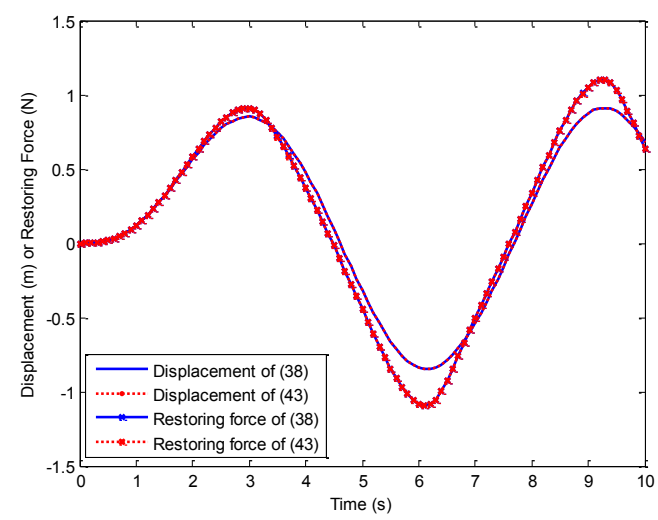

Fig. 6. Temporal behavior of hysteresis loops of (38) and (43).

Fig. 5 compares the hysteresis loops $(s, R)$ of the nonsmooth model (38) and the proposed linear model (43), where $e(t)=\sin (t), m=1, c=1, \alpha=0.5, k=1, D=1, \lambda=1, \beta=1, \gamma=$ $-1.5, n=2$, and the proposed model (43) with the approximation order $j=50$ is updated at a period of $0.2 \mathrm{~s}$. The temporal behaviors of displacement and restoring force are shown in Fig. 6. The loops and temporal curves from the proposed linear model (43) agree well with those from the non-smooth model (38), which verifies the effectiveness of the proposed method.

\section{CONCLUSION}

This paper proposes an innovative methodology to linearize a sign-function-constrained non-smooth model with the approximated scalar sign function. Through the proposed method, the non-smooth model can be transformed into a universal and smooth model. Thus, the following optimal linearization can be applied to obtain the local linear model at each operating point. Since traditional piecewise models are avoided, the resulting controller can be synthesized in a unified framework. The effectiveness of proposed method is demonstrated through two examples, representing typical non-smooth nonlinearities of friction and hysteresis, respectively.

\section{REFERENCES}

[1] M. Bernard, C. J. Budd, A. R. Champneys, and P. Kowalczyk, Piecewise-smooth Dynamical Systems, Theory and Applications. London, UK: Springer, 2008.

[2] H. J. Shieh and P. K. Huang, "Precise tracking of a piezoelectric positioning stage via a filtering type sliding surface control with chattering alleviation," IET Contr. Theo. and Appl., vol. 1, no. 3, pp. $586-594,2007$.

[3] J. D. Roberts, "Linear model reduction and solution of the algebraic Riccati equations by use of the sign function," Intl. Jour. Contr., vol. 32, pp. $677-687,1980$.

[4] C. S. Kenney and A. J. Laub, "The matrix sign function," IEEE Trans. Autom. Contr., vol. 40, no. 8, pp. 1330 - 1348, 1995.

[5] L. S. Shieh, Y. T. Tsay, and R. Yates, "Some properties of matrix sign functions derived from continued fractions," IEE Proc. Contr. Theo. and Appl., Part D, vol. 130, no. 3, pp. $111-118,1983$.

[6] F. Esfandiari and H. K. Khalil, "Output feedback stabilization of fully linearizable systems," International Journal of Control, vol. 56, pp. 1007-1037, 1992.

[7] M. C. M. Teixeira and S. H. Zak, "Stabilizing controller design for uncertain nonlinear systems using fuzzy models," IEEE Transaction on Fuzzy Systems, vol. 7, pp. 133-142, 1999.

[8] S. M. Guo, L. S. Shieh, G. Chen, and C. F. Lin, "Effective chaotic orbit tracker: a prediction-based digital redesign approach," IEEE Transaction on Circuits and Systems, vol. 47, pp. 1557-1570, 2000.

[9] G. Tao and F. L. Lewis, Adaptive Control of Non-smooth Dynamic Systems. London, UK: Springer-Verlag, 2001.

[10] R. Bouc, "Forced vibration of mechanical systems with hysteresis," in Proc. 4th Conf. Nonlinear Oscillation, Prague, Czechoslovakia, 1967, pp. 315.

[11] Y. K. Wen, "Method for random vibration of hysteretic systems," Jour. Engineering Mechanics Division, pp. 249 - 263, 1976.

[12] F. Ikhouane and J. Rodellar, Systems with Hysteresis: Analysis, Identification and Control using the Bouc-Wen Model. New York: Wiley, 2007.

[13] M. Ismail, F. Ikhouane, and J. Rodellar, "The hysteresis Bouc-Wen model, a survey," Arch. Comput. Methods Eng., vol. 16, pp. 161-188, 2009. 